\title{
Your Data, Your Vis: Personalizing Personal Data Visualizations
}

\author{
Hanna Schneider, Katrin Schauer, Clemens Stachl, and Andreas Butz \\ LMU Munich, Germany
}

\begin{abstract}
Personal Visualizations (PV) provide visual feedback on personal data, e. g., regarding physical activity or energy consumption. They are a vital part of many behavior change technologies (BCT) and Personal Informatics tools. Feedback can be presented in various ways, for example using counts and graphs, stylized displays, metaphoric displays, narrative information, data physicalisations, or even living plants. The properties of a PV are likely to influence its effectiveness towards reaching a goal. However, users' perceptions and preferences regarding different PVs seem to vary strongly, rendering a one-size-fits-all approach unsuitable. To investigate whether preferences for certain PVs coincide with personality or gender, we conducted a lab study with three example PVs: Donut, Glass, and Creature. Indeed, the results of our lab study are a first indicator that there is a relationship between personality traits and preferences for different PVs. High scores on extraversion and openness, for example, positively correlated with a preference for Creature. In contrast, high scores in conscientiousness negatively correlated with a preference for Creature. Further research is necessary to better understand how truly personalized PVs can be realized, which, in turn, might fit better into people's lives and thereby be more effective.
\end{abstract}

Keywords: Personal Informatics; visualization; personality; behavior change.

\section{Introduction}

Collecting and tracking personal data is becoming increasingly popular in various domains, ranging from physical activity (e.g., FitBit) to sleep (e.g., SleepCycle), food and water consumption (e.g., MyFitnessPal), and financial expenses (e.g., Mint) [18]. Tracking personal data can support people in becoming more aware of their habits and behaviors, changing their behavior, or reaching specific goals (such as spending less money or being more physically active) [8]. These systems are often called behavior change technologies (BCT) [25]. An important part of many BCTs is the way in which they present feedback, often delivered through personal visualizations $(\mathrm{PVs})$. One of the main challenges that $\mathrm{PVs}$ face is the diverse personalities and contexts that they should cater for [26]. As an example, for some users direct feedback, e.g., on the amount of weight gained or the amount of energy consumed, might induce negative feelings such as guilt or shame, while other users might appreciate the honesty of the technology and feel 
motivated. Several studies have reported that PVs provoked negative feelings among some users $[28,39]$. These negative experiences are named as a reason for the limited success of persuasive technologies, particularly in the long run [6]. One way to address this problem is to adapt the design of PVs to the individual needs and preferences of a user.
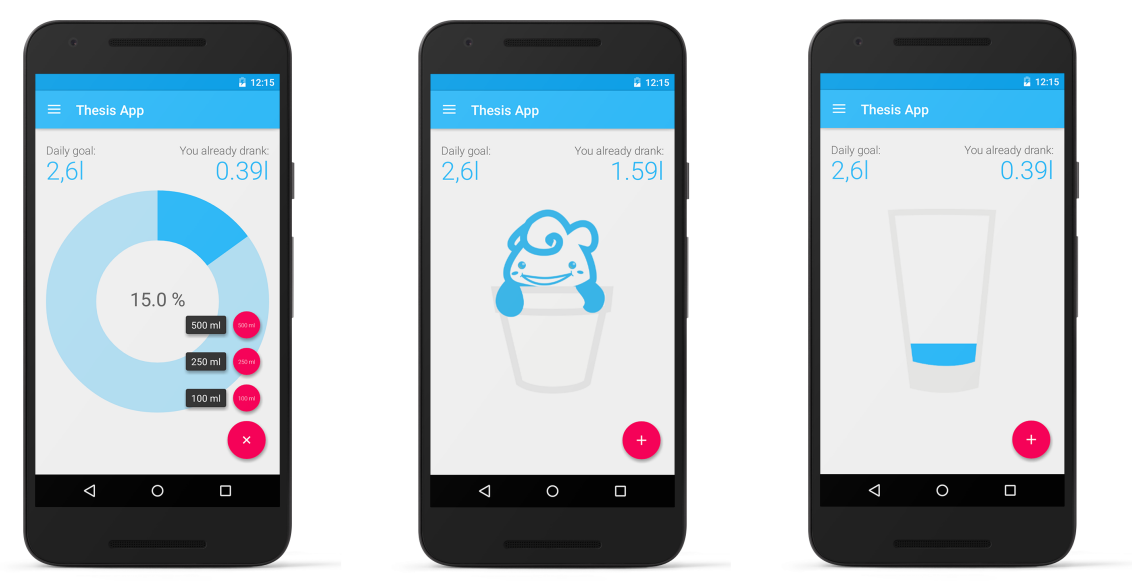

Fig. 1. Screenshots of the Donut (left), Creature (middle) and Glass (right) personal data visualization in the liquid-intake tracking application developed for our study.

In this work, we therefore tried to gain a better understanding of how preferences for PVs correlate with stable, well-researched personal traits based on the established Big Five personality theory. We developed three sample visualizations to convey users' daily liquid intake based on a review of existing liquid intake tracking applications: a plain visualization (Donut),and two decorated visualizations - namely a visualization based on the metaphor of a glass (Glass), and a visualization based on the metaphor of a creature (Creature) as depicted in fig. 1. We chose liquid intake as an example tracking metric because a broad range of users can relate to this use case. In a lab study, 36 participants were introduced to the three visualizations, conducted a personality test, and rated each visualization with respect to its attractiveness, its motivating or deterring effect, and their interest in its state.

Our hypothesis was that depending on the user's personality visualizations that make use of metaphors such as Creature and Glass might either be perceived as more engaging or deterring. Our results support this hypothesis. This paper reports the correlations between preferences for visualizations and personality traits and discusses directions for future research. 


\section{Related Work}

\subsection{Personal Visualizations (PVs)}

Huang et al. [26] defined the field of personal visualizations (PV) as "the design of interactive visual data representations for use in a personal context". Examples for PVs range from counts, graphs, and tables [11,18] via stylized displays [12,30], watchfaces [21] and narrative information [40] to data physicalisations [38] and living plants [5]. However, the suitability of different PVs for different contexts, goals, or users has rarely been systematically explored with a few exceptions: Epstein et al. [18] investigated how well different "visual cuts" allow users to explore their location and physical activity data. The visual cuts in their study were tables, graphs, captures, and maps. Because visualizations that people valued varied dramatically, Epstein et al. [18] recommended using a machine learning approach to offer users more effective or appropriate visualizations.

Choe et al. [7] and Eikey et al. [17] focused on persuasive performance feedback and investigated the influence of several design choices in health applications on self-efficacy. More specifically, Choe et al. [7] varied the valence of feedback (displaying the steps remaining to reach daily goal vs. displaying the achieved steps) and measured users' self-efficacy. They concluded that the achieved framing positively impacted participants' self-efficacy. Eikey et al. [17] varied the effect of color and distance to a goal and measured users' self-efficacy for accomplishing a goal. While they did not find significant results for color choice, they concluded that the remaining distance to a goal influenced users' self-efficacy.

While these studies revealed interesting results about the design of progress bars and text feedback, our study relates users' personalities and gender to their preferences for plain (Donut) and decorated visualizations (Glass, and Creature). Several researchers have explored potential advantages of decorative elements in charts, including for example memorability of charts, as examined by both Bateman et al. [4] and Li et al. [31]. While Bateman [4] concluded that visualizations with decorative elements were more memorable and preferred by most participants, Li [31] presented more ambiguous results: Some people perceived the decorative elements as inefficient, unprofessional and overloaded, while others perceived them as cute, attractive, and interesting. However, we do not yet understand, which users prefer what kind of visualization and in what context.

There is a lack of studies looking at users' preferences for decorative elements in PVs with respect to their individual differences. However, the idea of adapting visualizations has been applied to other types of visualizations under the name of user-adaptive visualizations.

\subsection{User-adaptive Information Visualization}

Several researchers (e. g., $[9,10,23,46])$ investigated the idea of user-adaptive visualizations, which are visualizations tailored in real-time to the needs and abilities of each user [9]. Existing work in this area has investigated the influence 
of both cognitive abilities $[10,41]$ and personality traits $[22,46]$ on the effectiveness of visualizations. Early results support the hypothesis that adapting visualizations to individual differences increases performance and satisfaction with visualizations significantly. However, existing studies focused on expert visualizations with the goal to extract complex information as quickly as possible. In contrast, the goal of the PVs used in our study is to motivate users to monitor their own data and work towards a goal. As discussed by Huang et al. [26], the nature of PVs is very different from expert visualizations. We are not aware of any studies that investigate how to adapt PVs to users' personality to motivate them towards reaching a goal.

\subsection{The Influence of Personality}

One possible source of influence on the perception of different types of data visualizations roots in users' personality dispositions. In psychological science, the dominant personality theory is the lexically derived Big Five personality trait theory $[13,20]$. In this theory personality is described by five broad traits, each of which consists of six sub-facets representing more narrow aspects of the personality. Below, we briefly summarize the Big Five:

People scoring high on extraversion are more sociable, lively, risk-taking, dominant, and in general prefer being in company with others over being alone. People scoring low on this personality trait are usually described as more reserved, reflected, and do not find much pleasure in spending time with many people [3]. People scoring high on emotional stability experience less negative emotions, feel more evenly-tempered, less impulsive, and also less responsive to stress. Low scores in emotional stability correspond to more negative feelings and longer durations of those [2]. Conscientiousness is a personality trait that describes how dutiful, self-disciplined, organized and deliberate someone is [32]. People scoring low on this dimension are typically more laid-back, less urged towards achievement, less goal-oriented, and less driven by success. This personality trait is closely related to academic as well as work place performance, and is therefore often assessed in job interviews [45]. The personality trait of Agreeableness is important for inter-personal relationships and describes how kind, sympathetic as well as helpful and considerate someone is. People with low scores usually are not very concerned about the feelings of people and are less likely to help others generally preferring competition over cooperation [27]. Openness is the personality trait that in general describes how curious someone is towards new experiences, feelings and attitudes. People with low values on this trait are generally described as more traditional, conservative and less flexible in their thinking and behavior. Openness is also correlated with intelligence and creativity [15].

We assume that users' preference for certain PVs might correlate with certain personality traits and sub-facets 


\section{Prototype}

For our lab study, we chose liquid intake as an example for self-tracking because the recommendation to drink a certain amount of liquid every day is wide-spread and well-known. Moreover, liquid volume is a simple metric to represent.

Instead of developing our own visualizations, we wanted to pick up state-ofthe-art visualizations that are already being used in applications today. Hence, we reviewed applications in app stores and found three broader visualization categories: those using plain charts and graphs, those using non-living metaphors (such as glasses and bottles) and those using living metaphors (such as plants and animals). As depicted in figure 2, we picked a plain visualization (Donut) and a living metaphor (Creature) as the two ends of a spectrum as we expected that users' personality might influence their preference for one or another. We added a non-living metaphor (Glass) as a third visualization to see whether it would be perceived similarly to the living metaphor.
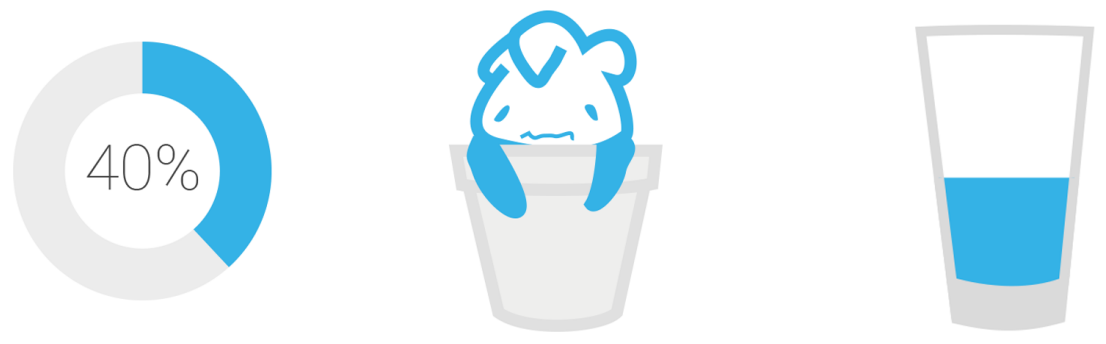

Fig. 2. Sketches of the three different concepts for visualizing liquid intake status compared in our study (left: Donut, middle: Creature, right: Glass).

\subsection{Donut Chart}

Donut is based on PVs often used by commercial tracking software such as Fitbit ${ }^{1}$, Jawbone $\mathrm{Up}^{2}$, Withings ${ }^{3}$, or the water consumption tracker "Hydrate Daily". Before any liquid intake was recorded, the circular bar chart is completely grey. When the user adds predefined amounts of liquid to the counter, the blue bar grows clockwise, filling up the circular bar to represent the user's progression towards the daily liquid goal (see figure 2 left).

\subsection{Creature Metaphor}

Creature makes use of the metaphor of a living plant. Empathy and compassion can be strong motivators and might therefore be used to foster behavior change [19].

\footnotetext{
1 https://www.fitbit.com

${ }^{2}$ https://jawbone.com/up

3 http://www.withings.com/
} 
There is evidence that users can emotionally engage with artificial pets and in many cases these emotions have therapeutic effects [34]. Botros et al. [5] used this effect by linking the well-being of a real plant to the physical activity of a user. Related, Creature displays a creature that suffers and is afraid to die of thirst when users themselves do not drink enough water. When a user adds predefined amounts of liquid, the creature becomes happier to represent the user's progression towards the daily liquid goal (see figure 3). Hence, the user is now responsible for the well-being of another virtual being. Several apps in the app store such as "Plant Nanny" use a similar concept.
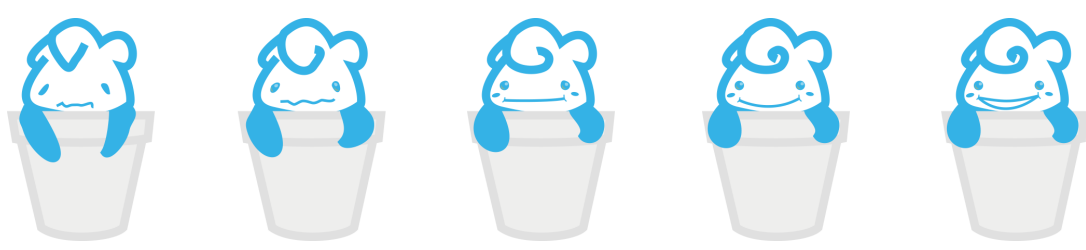

Fig. 3. Creature visualizes a user's intake of predefined liquid amounts with its progressing states of happiness.

\subsection{Glass Metaphor}

To allow the comparison between living and non-living metaphors, we introduced Glass, as shown in figure 2 right. It uses the metaphor of a glass, just as several apps in the app stores do (e.g., "Daily Water Free"). Again the water level (similar to the blue bar in Donut) fills up with the percentage of the daily liquid goal that has already been consumed.

\section{Method}

We ran a mixed between-within-subjects design with a 3 Concepts (Donut vs. Glass vs. Creature) $\times 2$ Gender (male vs. female) design and counterbalanced Concepts with a Latin Square for each Gender. We chose a lab setting for this initial exploration (instead of a field study) to reduce the influence of the context of use on our results.

\subsection{Evaluation Metrics}

As Huang et al. [26] pointed out, the evaluation of PVs presents a new challenge because typical visualization metrics (time and error rate) do not suffice and no best practices exist. They suggest "ease" as a new metric that defines how easily a tool fits into one's daily life, habits, and routine. "Ease" can, therefore, only be 
measured in long-term studies, in which participants use the PV in their daily life. Even though this is an important aspect of PV evaluations, we wanted to measure immediate aspects of the user experience that influence whether users feel inclined to use a PV again. Guided by literature on effects of design and information presentation on engagement $[24,35]$, motivation $[14,38]$, and self-efficacy $[7,17]$, we decided to use attractiveness, motivating effect, interest in its state, and deterring effect as dependent variables. To measure the dependent variables, we asked participants to express their agreement to the following statements on 7-point likert scales (statements translated from German):

attractiveness: "I perceive the visualization as attractive."

motivating effect: "I feel motivated to drink more water after looking at the visualization."

interest in its state: "I'm interested in the state of the visualization. I would look at it throughout the day."

deterring effect: "I perceive the visualization as deterring."

In Addition, we included three questions to validate whether all data visualizations and tasks were easy to understand.

\subsection{Task}

The purpose of the study task was that participants understood the three distinct concepts for visualizing liquid intake goals. Therefore, we asked participants to add predefined amounts of liquid to the liquid intake counter using the buttons provided and to verbalize their thoughts and questions using a thinkaloud technique. The researcher who conducted the experiment took notes of all comments and questions. Afterwards, participants were asked whether they understood the concept of the visualizations and any remaining questions were clarified. This procedure took between 3 and 10 minutes for each visualization.

\subsection{Personality Test}

We measured participants' personality traits using the Big Five Personality Inventory (BFSI) [1] in a laboratory setting. Performing the test took 18 minutes on average. We selected the BFSI for personality assessment due to its short length as well as its favorable psychometric properties.

\subsection{Procedure}

Our study set-up consisted of seven main steps: (1) informed consent (2) exploring the interactive prototype, (3) individual rating of each concept, (4) enforced ranking of all three concepts, (5) post-questionnaire about smart-phone usage, attitude towards tracking liquid intake, and demographics, and (6) personality test. Finally, (7) participants had the chance to express their opinions verbally, comments were noted down, clustered by researchers and considered in the analysis in addition to quantitative results. We used a unique identifier to link the different anonymized data types from questionnaires and personality test. 


\subsection{Participants}

We recruited 36 paid (7.50€) participants (18 female) through university mailing lists and social networks. Most participants were bachelor, master and $\mathrm{PhD}$ students with $44 \%$ studying Computer Science or similar subjects. Almost all participants (94\%) were smart-phone users with $45 \%$ having used smart-phones for at least three years. The majority of participants $(72,2 \%)$ had "tried to drink a certain amount of liquid every day before" confirming the relevance of the chosen scenario.

\subsection{Data Analysis}

To examine all possible relationships between personality, gender and preferences, we took an exploratory approach in our study design and data analysis. Due to the exploratory nature of our study, we report 95\% confidence intervals (CI) instead of p-values [36]. Confidence intervals not only provide information about the estimated range of the true values in the population (e.g., with repeated measures the CI would contain the correlation value of the population in $95 \%$ of the cases), but they also inform about significance in hypothesis testing research (not significant if the CI contains zero). In other words, CIs inform about the precision of the obtained estimator (narrow is good, wide is bad). These values inform hypotheses to be tested in future studies and should not readily be interpreted as established facts. Since we measured preferences for visualizations in ordinal scores, we used Spearman correlations to analyse correlations between ratings and personality dimensions [44]. Since scores on personality dimensions were normally distributed for both genders in our sample, we used Pearson product-moment correlation for correlations between predictor variables (see figure 4).

\subsection{Limitations}

Our study design has the following limitations: (1) Personality is a very complex phenomenon to study. Hence, it needs more than one study to build up an understanding of how individual differences are connected with preferences for visualizations. This study, therefore, can only present a starting point. As the study took on average 45 minutes to complete, we had to limit the number of PVs to three and restrict the amount of participants $(\mathrm{N}=36)$. Due to these restrictions, correlations have to be interpreted with caution. More example PVs need to be tested to be able to generalize our results. However, given that many correlation coefficients feature medium effect sizes (above a threshold of .30) and given that many correlations are in line with psychological literature, we believe that the results provide first insights into the relationships between personality, gender and preferences for PVs.

(2) Arguably, the scales, underlying the visualizations we used in our study, differ in their nature: Donut and Glass are based on a donut and a bar chart and can therefore easily be used as linear scales. Using Creature as linear scale is more difficult as value differences can not be clearly mapped to pixels: small differences 
in the gesture and facial expression of the creature might have a smaller or bigger effect on its expression and are open to interpretation by their users. To avoid issues with just noticeable differences we implemented the three visualizations as ordinal scales in our study (users could only add predefined amounts of liquid with provided buttons and those amounts were mapped to distinct states). Although the focus of our study was to compare three general visualization ideas and not on how these visualizations precisely progress, we conducted a prestudy to confirm that the different states of Creature were readable and clearly ordered in the eye of potential users. Therefore, we are confident that the differences of underlying scales did not affect our results. Nevertheless, readers should be aware of the potentially different perceptions of the scales underlying our visualizations.

(3) In line with Eikey et al.'s [17] approach, we measured participants' perceptions via self-report as this research is in a too early state to measure actual behavior. As we measured perceived attractiveness and interest in the state of the visualizations, we think the results of our study could inform a broader set of personal visualization systems and therefore have an impact beyond behavior change. We chose a controlled lab experiment to ensure the validity of the personality test and to minimize the influence of other contextual factors. However, whether long-term preferences differ (or not) needs to be tested in a long-term study. This will also allow to verify effectiveness towards behavior change, the main goal PVs for behavior change.

\section{Results}

We will first describe correlations between predictor variables and overall ratings of PVs in descriptive statistics. Next, we will describe correlations between preferences and personality traits.

\subsection{Descriptive Statistics}

Personality and Gender. For completeness, we report correlations between predictor variables. There were no significant correlations between gender and the Big Five personality factors. However, there were correlations between several Big Five personality factors (as shown in figure 4). The highest correlation was observed between extraversion and emotional stability $(\rho=0.639, p<0.001)$. We calculated variance inflation factors (VIF) for both extraversion (VIF $=1.5$ ) and emotional stability (VIF $=2.1$ ). As the VIF was smaller than 4 in both cases, we proceeded with the analysis [16].

Overall Ratings of PVs. All three concepts received ratings of four or higher on attractiveness by a majority of participants (Donut: $75 \%$, Glass: $61.1 \%$, Creature: $75 \%$ ). Creature was most often perceived as motivating (Donut: $44.4 \%$, Glass: $52.8 \%$, Creature: $61.1 \%)$. At the same time, the motivating effect of Creature also seemed to be more controversial (Donut: $M=4.17, S D=1.46$, Glass: $M=4.58$, 


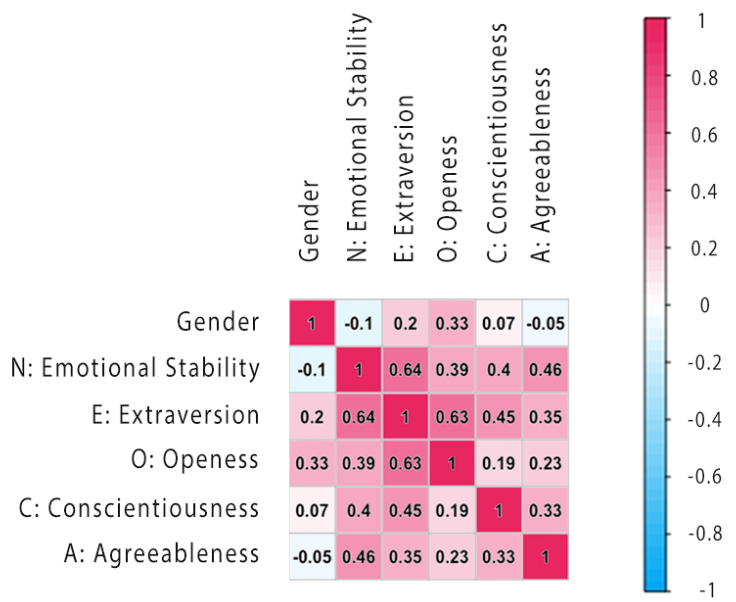

Fig. 4. Pairwise Pearson correlations between the predictor variables, namely, the Big Five measures and gender. Negative values mean a negative correlation, positive values a positive correlation. Gender is encoded as $0:=$ male, $1:=$ female.

$S D=1.40$, Creature: $M=4.67, S D=1.97)$. Moreover, more women perceived Creature as motivating than men (w: $72,2 \%, \mathrm{~m}: 50,0 \%$ ). The percentage of people interested in the PV's state was highest for Glass (Donut: $55.6 \%$, Glass: $72.2 \%$, Creature: $58.3 \%$ ). An equal number of men and women was interested in the state of Glass (72\%). All three concepts were perceived as deterring by only one participant: Donut and Glass by a female, and Creature by a male participant.

\subsection{Correlations between Preferences and Personality Traits}

Correlations between Likert scale ratings and the five main BFSI personality dimensions are visualized in figure 5 . Correlations with subdimensions are shown in figure 6.

We report correlations with a coefficient bigger than 0.2 , respectively smaller than -0.2 , in the text. Below, we first describe correlations between personality dimensions and Creature and Donut as these PVs were chosen as two ends of a spectrum. Then, we describe correlations with Donut, a PV that we added to contrast Creature with a non-living metaphor. Finally, we describe correlations with gender.

Creature Metaphor High scores in openness, extraversion, agreeableness, and emotional stability positively correlated with perceptions of Creature as attractive and motivating while high scores in conscientiousness correlated with perceiving Creature as deterring. More details on correlations between ratings of Creature and the main and subdimensions of personality, including $95 \%$ confidence intervals, are displayed in figure 7 . 


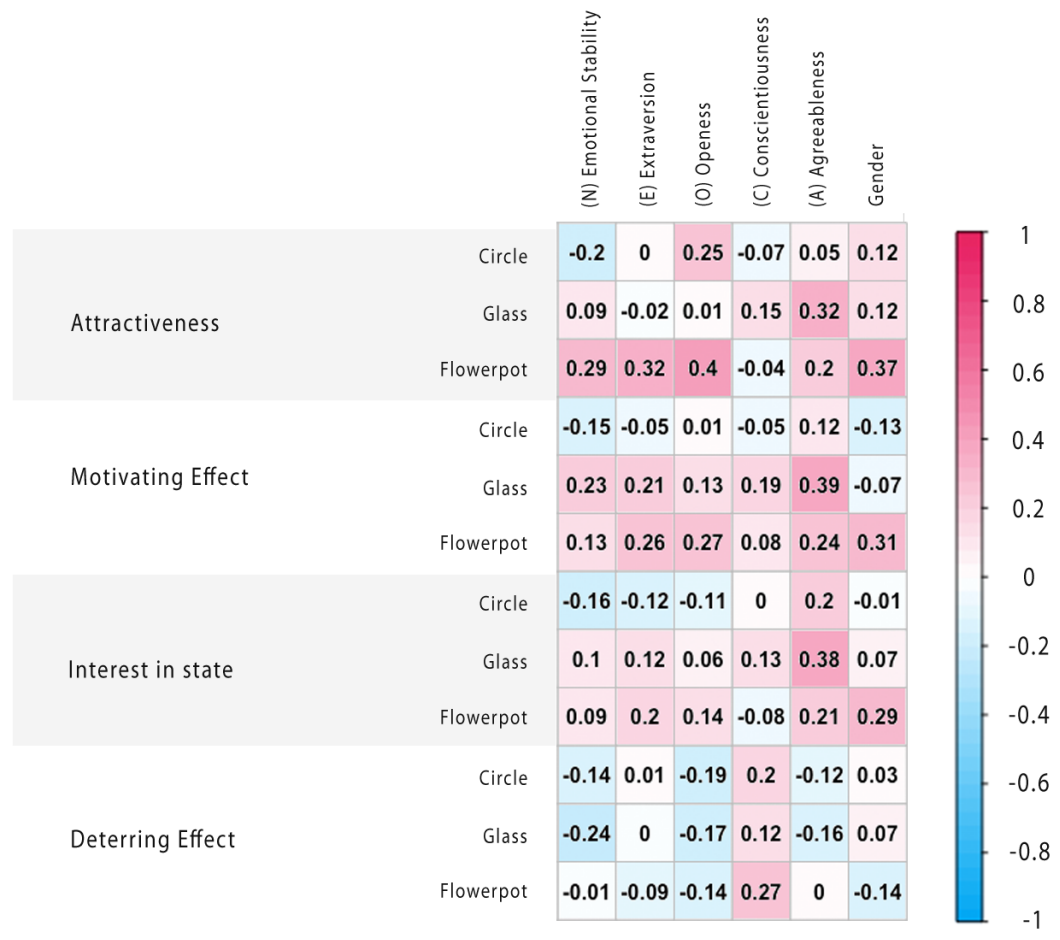

Fig. 5. Spearman's rank correlations: Participants rated the three concepts with regard to the criteria on the left on a seven-point Likert scale where 1:="I absolutely disagree", $7:=$ "I absolutely agree". The values of the Big Five dimensions were measured with the BFSI, gender is encoded as $0:=$ male, $1:=$ female.

Donut Chart Correlations between personality and ratings of Donut were generally very small. Only openness to aesthetics, a subdimension of openness, correlated with higher ratings of Donut as attractive.

Glass Metaphor Higher scores on agreeableness correlated with higher interest in the state of Glass, and with higher ratings of Glass as attractive and as motivating. Lower scores in emotional stability correlated with higher ratings of Glass as deterring. More details on correlations between ratings of Glass and the main and subdimensions of personality, including $95 \%$ confidence intervals, are displayed in figure 8 . 


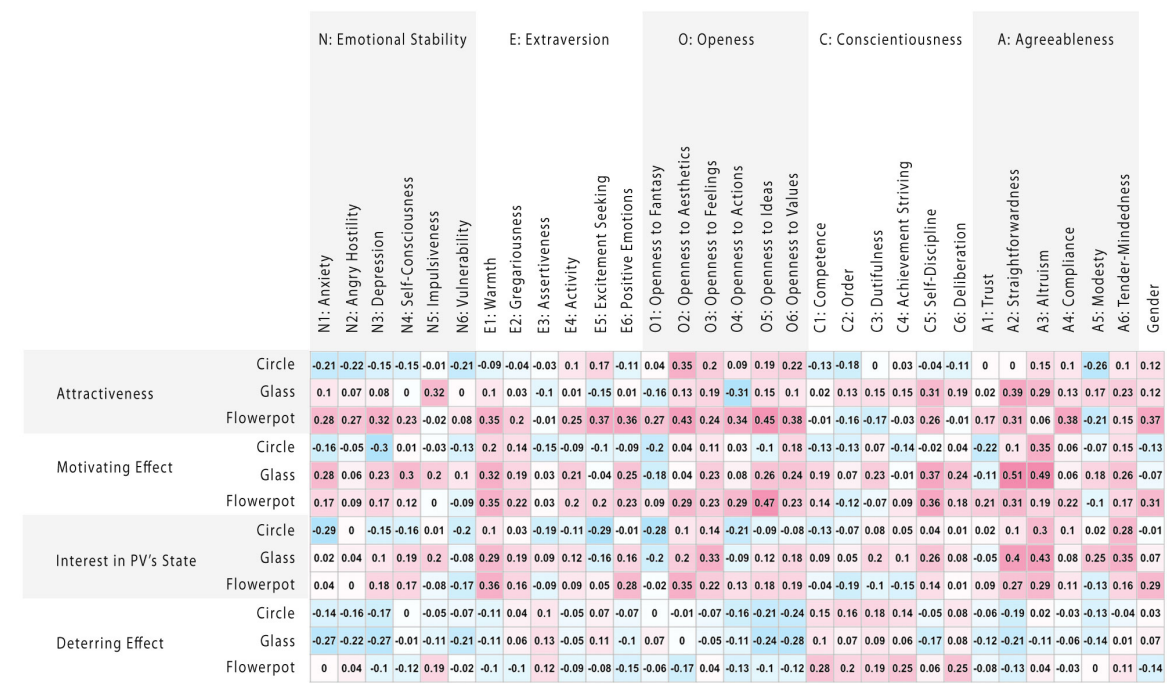

Fig. 6. Spearman's rank correlations: Participants rated the three concepts with regard to the criteria on the left on a seven-point Likert scale where 1:="I absolutely disagree", $7:=$ "I absolutely agree". The values of the subdimensions of the Big Five dimensions were measured with the BFSI, gender is encoded as $0:=$ male, $1:=$ female.

\subsection{Correlations with Gender}

Both women and men perceived Donut and Glass similarly. However, women rated Creature more often as attractive, motivating, and were more often interested in its state than men and than in the states of Glass and Donut.

\subsection{Qualitative Comments}

From qualitative comments and discussions in our study several alternative themes and metaphors for PVs emerged. Themes suggested by participants included rivers that drain or flood, ships that go aground or sail through heavy swell, and illustrations telling the story of physiological processes related to liquid intake. Moreover, some users in our study mentioned that they would prefer a visualization where the amount of liquid they consumed was subtracted of the daily goal rather than added up (remaining framing). However, as we did not collect preferences on the valence of feedback by all participants, we were not able to investigate if preferences coincide with users' personality or gender.

\section{Discussion and Future Work}

We see three main takeaways of our study: (1) It seems likely that preference and aversion for PVs coincide with users' personalities as measured with the Big Five 


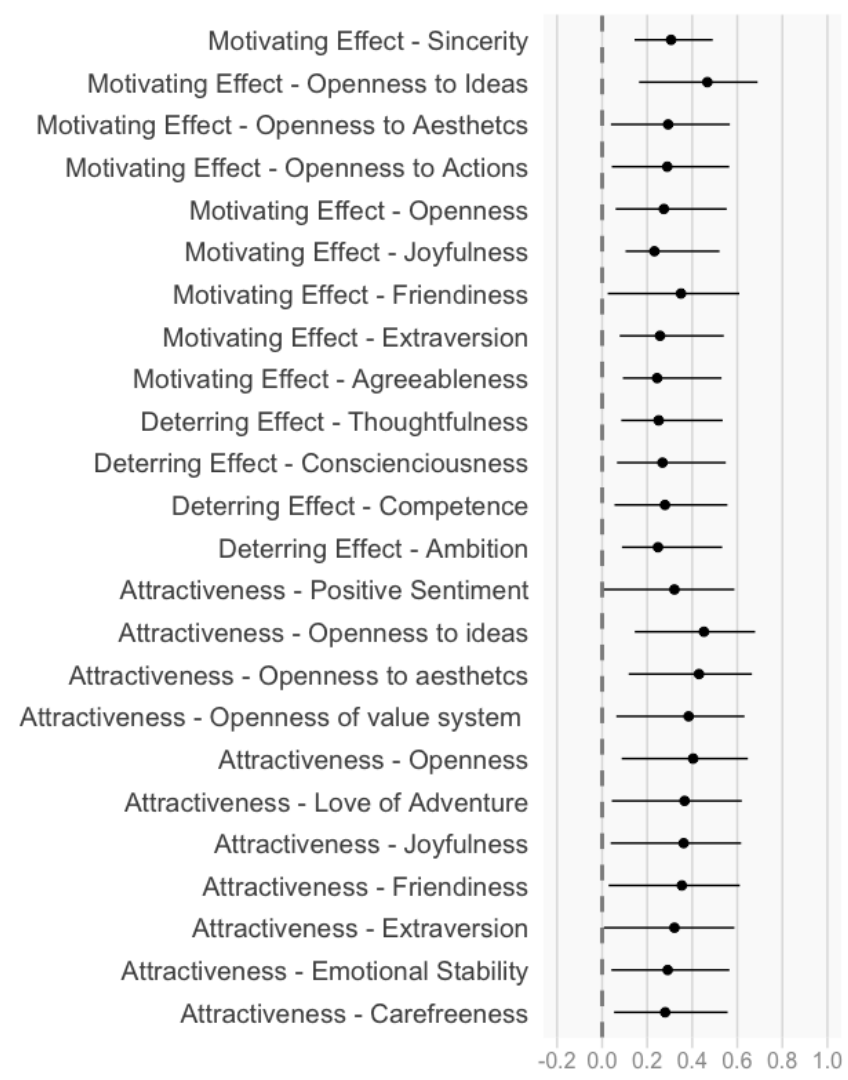

Fig. 7. Displaying the $95 \%$ confidence intervals of spearman correlations between ratings of Creature and personality dimensions

personality questionnaire. (2) To create PVs that are as engaging as possible, they need to be adapted to suit users preferences. This might be achieved in several ways. (3) Finally, our work provides guidance on how personalized PVs can be evaluated in future research.

\subsection{Personality and Preferences for PVs}

In our study, especially the personality traits openness (openness to new ideas, aesthetics, and openness of value system), extraversion (love of adventure, friendliness, and joyfulness), and agreeableness correlated with positive ratings of Creature. This observation is in line with previous research, that associated these personality traits with a need for affect [33]. People with a high need for affect tend to choose, for example, emotional movies or to become involved in an emotion-inducing event [33]. Hence, it seems plausible that related personality 


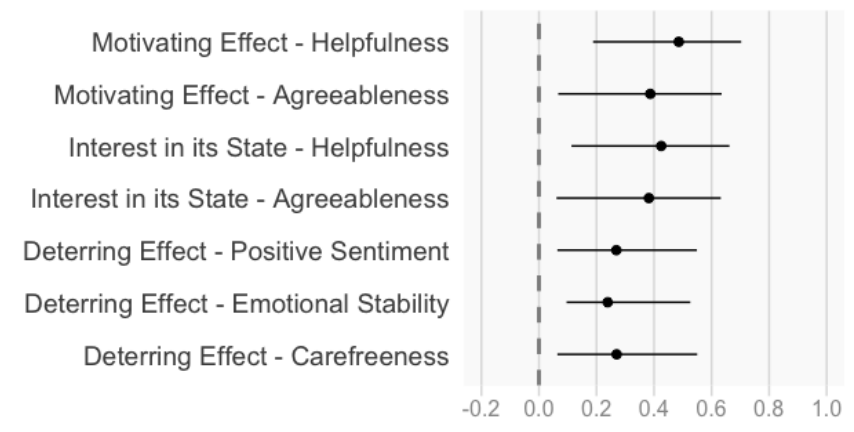

Fig. 8. Displaying the $95 \%$ confidence intervals of spearman correlations between ratings of Glass and personality dimensions

traits correlate with a preference for PVs, which make use of decorative elements and metaphors. Moreover, we found a tendency that conscientious people (described as dutiful, organized, self-disciplined, and deliberate) found Creature more deterring. In previous research conscientiousness was negatively associated with internet use [29] and playing videogames [43]. Hence, it seems plausible that more conscientious people are less drawn towards entertaining and decorating elements and prefer clean and plain visualizations. Correlations between perceptions of Glass and personality traits were somewhat similar but less and weaker than correlations with perceptions of Creature. Moreover, in our study, women tended to rate Creature more positively than Donut and Glass. These findings bring a new perspective to discussions of the usefulness of decorative elements in charts. It seems likely that such decorative elements can make PVs more engaging especially for users with a certain personality. However, future research is necessary validate these findings with more users and to explore a wider set of visualizations.

\subsection{Design Space of Personalized PVs}

We limited the set of PVs in our study to three, in order to keep the length of the experiment reasonable. These visualizations are examples taken from a rich design space. This design space encompasses, for example, different themes and metaphors not limited to plants and animals. Even for the relatively simple example of liquid intake visualizations, a variety of themes and metaphors emerged during our study in qualitative comments and discussions with participants. Future work should further analyze and explore this design space, e.g., in participatory design sessions and investigate how preferences are connected with users' individual differences. 


\subsection{Evaluation of Personalized PVs}

To explore the design space of PVs and the suitability of visualizations for different users and user groups, it is necessary to define evaluation criteria. Established evaluation metrics for visualizations - such as time and error rate - are, however, unsuitable for PVs. Rather than efficiency, the user experience is more likely to influence whether the PV is adopted and used over a longer period of time. In this study, we therefore used attractiveness, motivating effect, deterring effect, and interest in its state as evaluation metrics. However, even though these metrics allowed us to gain a first understanding of users' perceptions, they are tentative and most certainly need to be rethought in future studies. Beyond these, there are likely other relevant metrics, e. g., related to user experience or retention. If $\mathrm{PVs}$ are part of BCT actual behavior change is an important metric to measure in the long term. Future work should expand and explore the set of evaluation techniques used for PVs including the use of PVs in context and in the long term.

\subsection{Adaptive vs. Adaptable PVs}

Once a better understanding of the connection between users' individual differences and preferences for PVs is established, an important question to explore is how PVs could be adapted to better suit users' individual needs. As for all personalized systems, two general ways to achieve this personalization are to allow users to explicitly choose preferred visualizations (e.g., with a flexible framework that helps people design visualizations for themselves, as suggested by Huang et al. [26] or automatic adaptation. Automatic adaptation requires a user model that includes relevant characteristics of the user - in our case personality traits. These parameters might for example be inferred from mobile phone usage behaviors. Other trait-like factors such as cooperation have already been successfully predicted [37]. As data sets that contain both smartphone usage and personality data have already been collected from over 40.000 users, predicting personality traits based on mobile phone usage in the near future seems feasable [42]. However, some questions need to be answered before automatic adaptation becomes an option: When and how is a visualization adapted? How can adaptations be suggested without annoying or irritating the user?

\section{Conclusion}

There are many ways to visualize a user's personal data. PVs can be plain and direct like counts and graphs or more abstract like stylized displays or data physicalizations. Previous research showed that users seem to perceive different kinds of PVs very differently. To shed light on potential relationships between personality, gender, and preferences for PVs, we conducted an exploratory lab study with three example PVs. According to our results, preferences for PVs do indeed coincide with personality: For example, participants who scored high on openness and extraversion were more likely to prefer the PV with a living 
metaphor, while participants who scored high on conscientiousness rated it more negatively. Our results are in line with previous psychological research. Therefore, we are confident that our results provide promising insights into the relationships between personality and preferences for PVs. However, our research can not be more than a starting point at this stage and further validation of our findings is needed.

\section{References}

1. Arendasy, M.: BFSI: Big-Five Struktur-Inventar (Test \& Manual). Mödling: SCHUHFRIED GmbH. (2009)

2. Asendorpf, J.B., Neyer, F.J.: Psychologie der Persönlichkeit. Springer Berlin Heidelberg : Imprint: Springer, Berlin, Heidelberg (2012)

3. Ashton, M.C., Lee, K., Paunonen, S.V.: What is the central feature of extraversion? Social attention versus reward sensitivity. Journal of personality and social psychology 83(1), 245-252 (2002)

4. Bateman, S., Mandryk, R.L., Gutwin, C., Genest, A., McDine, D., Brooks, C.: Useful junk?: The effects of visual embellishment on comprehension and memorability of charts. In: Proceedings of the SIGCHI Conference on Human Factors in Computing Systems. pp. 2573-2582. CHI '10, ACM, New York, NY, USA (2010), http://doi. acm.org/10.1145/1753326.1753716

5. Botros, F., Perin, C., Aseniero, B.A., Carpendale, S.: Go and grow: Mapping personal data to a living plant. In: Proceedings of the International Working Conference on Advanced Visual Interfaces. pp. 112-119. AVI '16, ACM, New York, NY, USA (2016), http://doi.acm.org/10.1145/2909132.2909267

6. Brynjarsdottir, H., Håkansson, M., Pierce, J., Baumer, E., DiSalvo, C., Sengers, P.: Sustainably unpersuaded: How persuasion narrows our vision of sustainability. In: Proceedings of the SIGCHI Conference on Human Factors in Computing Systems. pp. 947-956. CHI '12, ACM, New York, NY, USA (2012), http://doi.acm.org/10. $1145 / 2207676.2208539$

7. Choe, E.K., Lee, B., Munson, S., Pratt, W., Kientz, J.A.: Persuasive performance feedback: The effect of framing on self-efficacy. In: AMIA Annual Symposium Proceedings. vol. 2013, p. 825. American Medical Informatics Association (2013)

8. Choe, E.K., Lee, N.B., Lee, B., Pratt, W., Kientz, J.A.: Understanding quantifiedselfers' practices in collecting and exploring personal data. In: Proceedings of the SIGCHI Conference on Human Factors in Computing Systems. pp. 1143-1152. CHI '14, ACM, New York, NY, USA (2014), http://doi.acm.org/10.1145/2556288. 2557372

9. Conati, C., Carenini, G., Toker, D., Lalle, S.: Towards user-adaptive information visualization (2015), http://www.aaai.org/ocs/index.php/AAAI/AAAI15/paper/ view/9933

10. Conati, C., Maclaren, H.: Exploring the role of individual differences in information visualization. In: Proceedings of the Working Conference on Advanced Visual Interfaces. pp. 199-206. AVI '08, ACM, New York, NY, USA (2008), http://doi. acm.org/10.1145/1385569.1385602

11. Consolvo, S., Klasnja, P., McDonald, D.W., Landay, J.A.: Designing for healthy lifestyles: Design considerations for mobile technologies to encourage consumer health and wellness. Foundations and Trends in Human-Computer Interaction 6(3-4), 167-315 (2014), http://dx.doi.org/10.1561/1100000040 
12. Consolvo, S., McDonald, D.W., Toscos, T., Chen, M.Y., Froehlich, J., Harrison, B., Klasnja, P., LaMarca, A., LeGrand, L., Libby, R., Smith, I., Landay, J.A.: Activity sensing in the wild: A field trial of ubifit garden. In: Proceedings of the SIGCHI Conference on Human Factors in Computing Systems. pp. 1797-1806. CHI '08, ACM, New York, NY, USA (2008), http://doi.acm.org/10.1145/1357054.1357335

13. Costa, P.T., McCrae, R.R.: Four ways five factors are basic. Personality and Individual Differences 13(6), 653 - 665 (1992), http://www.sciencedirect.com/ science/article/pii/019188699290236I

14. Deci, E.L.: The relation of interest to the motivation of behavior: A selfdetermination theory perspective. (1992)

15. DeYoung, C.G.: Openness/intellect: A dimension of personality reflecting cognitive exploration. In: Mikulincer, M., Shaver, P.R., Cooper, M.L., Larsen, R.J. (eds.) APA handbook of personality and social psychology, Volume 4: Personality processes and individual differences, pp. 369-399. APA handbooks in psychology., American Psychological Association, Washington, DC, US (2015)

16. Dormann, C.F., Elith, J., Bacher, S., Buchmann, C., Carl, G., Carré, G., Marquéz, J.R.G., Gruber, B., Lafourcade, B., Leitão, P.J., Münkemüller, T., McClean, C., Osborne, P.E., Reineking, B., Schröder, B., Skidmore, A.K., Zurell, D., Lautenbach, S.: Collinearity: a review of methods to deal with it and a simulation study evaluating their performance. Ecography 36(1), 27-46 (2013), http://dx.doi.org/10.1111/j.16000587.2012.07348.x

17. Eikey, E., Poole, E., Reddy, M.: Information presentation in health apps and devices: the effect of color, distance to goal, weight perception, and interest on users' self-efficacy for accomplishing goals. iConference 2015 Proceedings (2015)

18. Epstein, D., Cordeiro, F., Bales, E., Fogarty, J., Munson, S.: Taming data complexity in lifelogs: Exploring visual cuts of personal informatics data. In: Proceedings of the 2014 Conference on Designing Interactive Systems. pp. 667-676. DIS '14, ACM, New York, NY, USA (2014), http://doi.acm.org/10.1145/2598510.2598558

19. Goetz, J.L., Keltner, D., Simon-Thomas, E.: Compassion: an evolutionary analysis and empirical review. Psychological bulletin 136(3), 351 (2010)

20. Goldberg, L.: Language and individual differences: The search for universals in personality lexicons. In: Review of Personality and Social Psychology, vol. 2, pp. 141-165. Beverly Hills, CA (1981)

21. Gouveia, R., Pereira, F., Karapanos, E., Munson, S.A., Hassenzahl, M.: Exploring the design space of glanceable feedback for physical activity trackers. In: Proceedings of the 2016 ACM International Joint Conference on Pervasive and Ubiquitous Computing. pp. 144-155. UbiComp '16, ACM, New York, NY, USA (2016), http: //doi.acm.org/10.1145/2971648.2971754

22. Green, T.M., Fisher, B.: Towards the personal equation of interaction: The impact of personality factors on visual analytics interface interaction. In: Visual Analytics Science and Technology (VAST), 2010 IEEE Symposium on. pp. 203-210. IEEE (2010)

23. Green, T.M., Fisher, B.: Impact of personality factors on interface interaction and the development of user profiles: Next steps in the personal equation of interaction. Information Visualization 11(3), 205-221 (Jul 2012), http://dx.doi.org/10.1177/ 1473871612441542

24. Haroz, S., Kosara, R., Franconeri, S.L.: Isotype visualization: Working memory, performance, and engagement with pictographs. In: Proceedings of the 33rd annual ACM conference on human factors in computing systems. pp. 1191-1200. ACM (2015) 
25. Hekler, E.B., Klasnja, P., Froehlich, J.E., Buman, M.P.: Mind the theoretical gap: Interpreting, using, and developing behavioral theory in hci research. In: Proceedings of the SIGCHI Conference on Human Factors in Computing Systems. pp. 3307-3316. CHI '13, ACM, New York, NY, USA (2013)

26. Huang, D., Tory, M., Aseniero, B.A., Bartram, L., Bateman, S., Carpendale, S., Tang, A., Woodbury, R.: Personal visualization and personal visual analytics. IEEE Transactions on Visualization and Computer Graphics 21(3), 420-433 (March 2015)

27. Jensen-Campbell, L., Graziano, W.G.: Agreeableness as a moderator of interpersonal conflict. Journal of personality 69(2), 323-361 (2001)

28. Kuznetsov, S., Paulos, E.: Upstream: Motivating water conservation with lowcost water flow sensing and persuasive displays. In: Proceedings of the SIGCHI Conference on Human Factors in Computing Systems. pp. 1851-1860. CHI '10, ACM, New York, NY, USA (2010), http://doi.acm.org/10.1145/1753326.1753604

29. Landers, R.N., Lounsbury, J.W.: An investigation of big five and narrow personality traits in relation to internet usage. Computers in Human Behavior 22(2), $283-$ 293 (2006), http://www.sciencedirect.com/science/article/pii/S0747563204001128

30. Lane, N.D., Lin, M., Mohammod, M., Yang, X., Lu, H., Cardone, G., Ali, S., Doryab, A., Berke, E., Campbell, A.T., Choudhury, T.: Bewell: Sensing sleep, physical activities and social interactions to promote wellbeing. Mob. Netw. Appl. 19(3), 345-359 (Jun 2014), http://dx.doi.org/10.1007/s11036-013-0484-5

31. Li, H., Moacdieh, N.: Is "chart junk" useful? an extended examination of visual embellishment. Proceedings of the Human Factors and Ergonomics Society Annual Meeting 58(1), 1516-1520 (2014), http://pro.sagepub.com/content/58/1/1516.abstract

32. MacCann, C., Duckworth, A.L., Roberts, R.D.: Empirical identification of the major facets of Conscientiousness. Learning and Individual Differences 19(4), 451-458 (2009)

33. Maio, G.R., Esses, V.M.: The need for affect: Individual differences in the motivation to approach or avoid emotions. Journal of Personality 69(4), 583-614 (2001), http://dx.doi.org/10.1111/1467-6494.694156

34. Marti, P., Pollini, A., Rullo, A., Shibata, T.: Engaging with artificial pets. In: Proceedings of the 2005 Annual Conference on European Association of Cognitive Ergonomics. pp. 99-106. EACE '05, University of Athens (2005), http://dl.acm. org/citation.cfm?id=1124666.1124680

35. Norman, D.: Emotion \& design: attractive things work better. interactions 9(4), 36-42 (2002)

36. Robert G. Jaeger, T.R.H.: On confirmatory versus exploratory research. Herpetologica 54, S64-S66 (1998), http://www.jstor.org/stable/3893289

37. Singh, V.K., Agarwal, R.R.: Cooperative phoneotypes: Exploring phone-based behavioral markers of cooperation. In: Proceedings of the 2016 ACM International Joint Conference on Pervasive and Ubiquitous Computing. pp. 646-657. UbiComp '16, ACM, New York, NY, USA (2016), http://doi.acm.org/10.1145/ 2971648.2971755

38. Stusak, S., Tabard, A., Sauka, F., Khot, R.A., Butz, A.: Activity sculptures: Exploring the impact of physical visualizations on running activity. IEEE Transactions on Visualization and Computer Graphics 20(12), 2201-2210 (Dec 2014)

39. Thieme, A., Comber, R., Miebach, J., Weeden, J., Kraemer, N., Lawson, S., Olivier, P.: "we've bin watching you": Designing for reflection and social persuasion to promote sustainable lifestyles. In: Proceedings of the SIGCHI Conference on Human Factors in Computing Systems. pp. 2337-2346. CHI '12, ACM, New York, NY, USA (2012), http://doi.acm.org/10.1145/2207676.2208394 
40. Tollmar, K., Bentley, F., Viedma, C.: Mobile health mashups: Making sense of multiple streams of wellbeing and contextual data for presentation on a mobile device. In: 2012 6th International Conference on Pervasive Computing Technologies for Healthcare (PervasiveHealth) and Workshops. pp. 65-72 (May 2012)

41. Velez, M.C., Silver, D., Tremaine, M.: Understanding visualization through spatial ability differences. In: VIS 05. IEEE Visualization, 2005. pp. 511-518. IEEE (2005)

42. Welke, P., Andone, I., Blaszkiewicz, K., Markowetz, A.: Differentiating smartphone users by app usage. In: Proceedings of the 2016 ACM International Joint Conference on Pervasive and Ubiquitous Computing. pp. 519-523. UbiComp '16, ACM, New York, NY, USA (2016), http://doi.acm.org/10.1145/2971648.2971707

43. Witt, E.A., Massman, A.J., Jackson, L.A.: Trends in youth's videogame playing, overall computer use, and communication technology use: The impact of self-esteem and the big five personality factors. Computers in Human Behavior 27(2), $763-$ 769 (2011), http://www.sciencedirect.com/science/article/pii/S0747563210003237, web 2.0 in Travel and Tourism: Empowering and Changing the Role of Travelers

44. Yarkoni, T.: Personality in 100,000 words: A large-scale analysis of personality and word use among bloggers. Journal of Research in Personality 44(3), $363-373$ (2010), http://www.sciencedirect.com/science/article/pii/S0092656610000541

45. Ziegler, M., Bensch, D., Maaß, U., Schult, V., Vogel, M., Bühner, M.: Big Five facets as predictor of job training performance: The role of specific job demands. Learning and Individual Differences 29, 1-7 (2014)

46. Ziemkiewicz, C., Kosara, R.: Preconceptions and individual differences in understanding visual metaphors. In: Proceedings of the 11th Eurographics / IEEE VGTC Conference on Visualization. pp. 911-918. EuroVis'09, The Eurographs Association; John Wiley and Sons, Ltd., Chichester, UK (2009), http://dx.doi.org/ 10.1111/j.1467-8659.2009.01442.x 